\title{
STRUCTURES GÉOMÉTRIQUES HOLOMORPHES SUR LES VARIÉTÉS COMPLEXES COMPACTES
}

\author{
PAR SORIN DUMITRESCU
}

ABSTRACT. - We study holomorphic geometric structures on compact complex manifolds. We show that, contrary to the situation in the real domain, a holomorphic geometric structure on a compact complex manifold usually admits a "big" pseudogroup of local isometries. We exhibit very general conditions which imply that the pseudogroup of local isometries acts transitively.

(C) 2001 Éditions scientifiques et médicales Elsevier SAS

RÉSUMÉ. - Nous étudions les structures géométriques holomorphes sur les variétés complexes compactes. Nous montrons que, contrairement au cas réel, une telle structure possède souvent un «grand» pseudogroupe d'isométries locales. Nous exhibons des conditions très générales qui garantissent l'existence d'une action transitive du pseudogroupe des isométries locales.

(C) 2001 Éditions scientifiques et médicales Elsevier SAS

\section{Introduction}

L'étude des structures géométriques sur les variétés différentiables doit beaucoup de son développement actuel à l'immense œuvre d'Élie Cartan.

Avant d'introduire la définition rigoureuse du concept de structure géométrique, précisons qu'un champ de tenseurs holomorphe, un champ de plans holomorphe, une connexion affine holomorphe, ou encore une structure symplectique holomorphe sont des cas particuliers (mais importants) de structures géométriques holomorphes.

Contrairement à la géométrie réelle, l'hypothèse de l'existence d'une structure géométrique holomorphe sur une variété complexe compacte est une condition très restrictive pour la variété.

Le but de cet article est de montrer que, contrairement au cas réel, une structure géométrique holomorphe sur une variété complexe compacte possède souvent un «grand»pseudogroupe d'isométries locales. Dans ce sens, nous exhibons des conditions très générales qui garantissent l'existence d'une action transitive du pseudogroupe des isométries locales.

Le sujet de cet article nous a été proposé par Étienne Ghys. Nous tenons à le remercier ici pour son aide précieuse et pour ses encouragements constants.

\subsection{Cadre géométrique}

Pour définir rigoureusement le concept plutôt abstrait de structure géométrique, il est agréable de se placer dans le cadre des structures infinitésimales créé par Charles Ehresmann à cet effet.

Associons à une variété complexe $M$ de dimension $n$ l'espace des germes en 0 de tous les biholomorphismes d'un ouvert de $\mathbf{C}^{n}$ qui contient 0 à valeurs dans un ouvert de $M$. Identifions 
deux éléments de cet espace s'ils ont le même $r$-jet en 0 (i.e. même polynôme de Taylor à l'ordre $r$ en 0). La légitimité de cette opération est assurée par le fait que la relation d'équivalence «avoir le même développement de Taylor en 0 » ne dépend pas du choix d'un système de coordonnées locales). Nous admettrons que les éléments du quotient précédent s'organisent en un fibré principal au-dessus de $M$ : le fibré des r-repères. Désignons-le par $R^{r}(M)$ et précisons que le fibré des $r$-repères est un fibré principal au-dessus de $M$ de groupe structural $D^{r}\left(\mathbf{C}^{n}\right)$, où $D^{r}\left(\mathbf{C}^{n}\right)$ est le groupe des $r$-jets en 0 des biholomorphismes locaux de $\mathbf{C}^{n}$ qui préservent 0. Ce groupe porte une structure naturelle de groupe algébrique. Par exemple, le fibré des 1-repères s'identifie avec l'ensemble des bases de tous les espaces tangents à la variété et $D^{1}\left(\mathbf{C}^{n}\right)$ n'est rien d'autre que $G L(n, \mathbf{C})$.

Par la suite nous allons considérer aussi l'espace des $r$-jets en 0 d'applications holomorphes d'un ouvert de $\mathbf{C}^{p}$ qui contient 0 à valeurs dans la variété $M$. Il s'agit d'une variété $J^{r, p}(M)$ qui fibre sur $M$, la projection étant simplement l'évaluation en 0 .

Pour des précisions supplémentaires sur la théorie des fibrés des jets nous référons à [21] et à [3].

La définition suivante coïncide avec le concept de $A$-structure introduit dans [13] par M. Gromov.

DÉFINITION 1.1. - Soit $Z$ une variété quasiprojective (i.e. un ouvert de Zariski d'une variété projective) munie d'une action algébrique de $D^{r}\left(\mathbf{C}^{n}\right)$. Une structure géométrique de type $Z$ et d'ordre $r$ sur la variété complexe $M$ est une application holomorphe $D^{r}\left(\mathbf{C}^{n}\right)$-équivariante $\phi: R^{r}(M) \rightarrow Z$, i.e. $\phi(s \cdot g)=g^{-1} \cdot \phi(s), \forall s \in R^{r}(M)$ et $\forall g \in D^{r}\left(\mathbf{C}^{n}\right)$.

Une structure géométrique s'interprète également comme une section d'un fibré naturel construit au-dessus de la variété.

Une structure géométrique est dite de type algébrique affine si la variété $Z$ est affine (par exemple, un champ de vecteurs est une structure géométrique de type algébrique affine, un champ de droites ne l'est pas).

Dans l'esprit de la définition précédente un champ holomorphe de formes quadratiques complexes sur l'espace tangent à $M$ est une application holomorphe $G L(n, \mathbf{C})$-équivariante du fibré des 1-repères à valeurs dans l'espace vectoriel $Z$ formée par les formes bilinéaires symétriques sur $\mathbf{C}^{n}$. L'action de $G L(n, \mathbf{C})$ sur $Z$ est l'action usuelle obtenue par changement de base.

Plus généralement, un champ de tenseurs holomorphe de type $(p, q)$ sur la variété $M$, qui est par définition une section holomorphe du fibré vectoriel $T M^{\otimes p} \otimes\left(T M^{*}\right)^{\otimes q}$, se présente à la lumière de la définition 1.1 comme une structure géométrique d'ordre 1 dont le type est la représentation $\left(\mathbf{C}^{n}\right)^{\otimes p} \otimes\left(\mathbf{C}^{n *}\right)^{\otimes q}$ de $G L(n, \mathbf{C})$ (où $\mathbf{C}^{n *}$ désigne la représentation duale de la représentation canonique).

Une connexion affine holomorphe sur une variété complexe de dimension $n$ s'exprime dans une carte locale par $n^{3}$ fonctions holomorphes à valeurs dans $\mathbf{C}$ : les célèbres coefficients de Christoffel $\Gamma_{i j}^{k}$. Le type de cette structure est l'ensemble des 0 -jets de germes de connexion : $Z=\left\{\Gamma_{i j}^{k}(0) \mid 1 \leqslant i, j, k \leqslant n\right\} \simeq \mathbf{C}^{n^{3}}$. La variété $Z$ est munie de l'action du groupe $D^{2}\left(\mathbf{C}^{n}\right)$ obtenue de la manière suivante : l'image d'un germe de connexion en 0 par le germe d'un biholomorphisme local de $\mathbf{C}^{n}$ qui préserve 0 est un autre germe de connexion en 0 dont le 0 -jet ne dépend que du 0 -jet de la connexion de départ et du 2-jet en 0 du biholomorphisme de transport. Pour plus de détails nous renvoyons à [3], [8].

Le jet d'ordre $s$ d'une structure géométrique $\phi$ d'ordre $r$ est une nouvelle structure géométrique d'ordre $s+r$. Si dans une carte locale de la variété $\phi$ est déterminée par une application à valeurs dans la variété $Z$, son jet d'ordre $s$, que nous notons $\phi^{(s)}$, s'exprime dans la même carte par le $s$-jet de l'application initiale. Formellement $\phi^{(s)}$ est une structure de type 
$J^{s, n}(Z)$, où $n$ désigne la dimension de la variété. Pour se convaincre que ceci se formalise bien et que le jet d'une structure de type algébrique affine est encore une structure de type algébrique affine nous référons à [3], [8].

Dans le cas particulier où la structure géométrique $\phi$ est telle que l'image de $\phi$ dans $Z$ est exactement une $D^{r}\left(\mathbf{C}^{n}\right)$-orbite, nous avons aussi la terminologie de $G$-structure, le groupe $G$ étant le stabilisateur dans $D^{r}\left(\mathbf{C}^{n}\right)$ de l'orbite en question. Dans ce cas $G$ est un groupe structural pour le fibré $R^{r}(M)$.

Par exemple, un champ de formes quadratiques complexes sur l'espace tangent à $M$ est une $G$-structure si le rang ne dépend pas du point.

Quand ce rang est en tout point maximal nous avons une métrique riemannienne holomorphe, qu'il convient d'envisager comme l'analogue complexe d'une métrique pseudo-riemannienne. En effet, de manière analogue à la géométrie pseudo-riemannienne, à une métrique riemannienne holomorphe il est associé une unique connexion (holomorphe) de Levi-Civita, des courbes géodésiques (les géodésiques sont des courbes holomorphes dont le vecteur tangent est parallèle) et un tenseur de courbure.

Parmi les structures géométriques nous allons parfois distinguer celles qui sont rigides dans le sens de M. Gromov [13] : une structure géométrique $\phi$ d'ordre $r$ est dite rigide s'il existe un entier l tel que tout $(r+l+1)$-jet de biholomorphisme local de $M$ qui préserve le $(l+1)$-jet de $\phi$ est entièrement détérminé par sa partie d'ordre $r+l$.

Exemples : une connexion affine holomorphe ou bien une métrique riemannienne holomorphe sont des structures géométriques holomorphes rigides (l'explication réside essentiellement dans le fait que, pour ces deux structures géométriques, une isométrie locale qui fixe un point est entièrement détérminée par sa différentielle au point fixe).

Si la variété $M$ est munie d'une structure géométrique $\phi$, nous conviendrons d'appeler isométrie locale de $\phi$ un biholomorphisme entre deux ouverts de $M$ qui préserve la structure $\phi$. L'ensemble des isométries locales $I s^{l o c}$ est un pseudogroupe pour la composition.

Deux points de $M$ sont dans la même orbite du pseudogroupe des isométries locales s'il existe des systèmes de coordonnées centrés en ces points dans lesquels les expressions de $\phi$ sont les mêmes.

Quand le pseudogroupe $I s^{l o c}$ agit transitivement sur $M$, la structure géométrique $\phi$ est dite localement homogène. Cela veut dire qu'il existe une unique forme locale pour la structure.

\subsection{Exemples et motivations}

Si le théorème classique de Darboux assure, dans le cas d'une structure symplectique, l'existence d'une action transitive du pseudogroupe des isométries locales, ceci est entièrement faux lorsqu'il s'agit d'une métrique riemannienne holomorphe. Dans ce dernier cas nous disposons d'un invariant local fort qui est la courbure.

Pour la suite de cet article, nous nous plaçons sur une variété complexe compacte connexe $M$ munie d'une structure géométrique holomorphe $\phi$ (en général, l'existence d'une telle structure n'est nullement assurée, mais impose des conditions restrictives sur $M$ ). Remarquons que tout invariant scalaire de la structure $\phi$ est constant sur $M$ en tant que fonction holomorphe définie sur une variété compacte.

Ceci suffit dans certains cas pour conclure à l'homogénéité locale de la structure. Considérons, par exemple, une métrique riemannienne holomorphe sur une surface complexe compacte $S$. Il n'est pas difficile de définir la notion de courbure sectionnelle (voir [8]) qui est une fonction holomorphe sur $S$. La compacité de $S$ implique que cette courbure sectionnelle est constante et, par conséquent, un argument classique montre que la métrique est localement homogène. 
Le théorème suivant, dû à Wang, constitue un deuxième exemple où la remarque précédente permet de classifier toutes les variétés complexes compactes dont le fibré tangent est holomorphiquement trivial (une trivialisation holomorphe du fibré tangent est un exemple particulier de $G$-structure holomorphe où $G$ vaut $I d)$ :

THÉORÈME 1.2 (Wang). - Soit $M$ une variété complexe compacte de dimension $n$ dont le fibré tangent est holomorphiquement trivial (i.e. il existe $n$ sections holomorphes du fibré tangent, en chaque point linéairement indépendentes). Alors $M$ est nécessairement un quotient d'un groupe de Lie complexe $G$ par un réseau cocompact $\Gamma$.

Le théorème précédent affirme, en particulier, qu'une trivialisation holomorphe du fibré tangent d'une variété complexe compacte est une structure géométrique localement homogène.

La preuve du théorème de Wang est facile, mais instructive. Considérons une famille $X_{1}, \ldots, X_{n}$, de champs de vecteurs holomorphes sur $M$ qui forment en chaque point une base de l'espace tangent. Les crochets de Lie de ces champs, pris deux à deux, sont :

$$
\left[X_{i}, X_{j}\right]=\sum_{k=1}^{n} c_{i j}^{k} X_{k} .
$$

Les fonctions $c_{i j}^{k}$ ainsi définies sont holomorphes et, par conséquent, constantes. Ceci montre que les champs $X_{i}$ forment une algèbre de Lie de dimension finie. D'après un résultat classique, dû à S. Lie, il s'agit de l'algèbre de Lie $\mathcal{G}$ des champs de vecteurs invariants à droite d'un groupe de Lie complexe connexe et simplement connexe $G$ agissant à gauche sur $M$. Cette action est nécessairement transitive et $M$ s'identifie au quotient de $G$ par un réseau cocompact $\Gamma$. Les translations à gauche de $G$ sont des biholomorphismes globaux de $G / \Gamma$ qui agissent sur $\mathcal{G}$ par la représentation adjointe. Ce qui montre qu'en général l'action précédente n'est pas isométrique. En revanche, dans une carte locale de $M$, donnée par une section locale de la projection $G \rightarrow G / \Gamma$, les translations à droite définissent des isométries locales. Le parallélisme est donc une structure localement homogène, qui n'est pas globalement homogène. Le lecteur habitué au langage des $G$-structures remarquera que dans le cas où $G$ n'est pas abélien la structure n'est pas intégrable.

En revanche, il existe des structures géométriques holomorphes sur des variétés compactes qui ne sont pas localement homogènes (même pas sur un ouvert dense) : la droite projective $P^{1}(\mathbf{C})$ munie de deux champs de vecteurs holomorphes linéairement indépendants fournit un exemple de variété complexe compacte qui possède une structure géométrique holomorphe non localement homogène. En effet, nous pouvons fabriquer l'invariant scalaire qui, au point $m$ de $P^{1}(\mathbf{C})$, vaut la valeur en $m$ de la fraction rationnelle «quotient» des sections en question. Cette fraction rationnelle n'est pas constante et elle sépare les orbites du pseudogroupe $I s^{l o c}$.

\section{3. Énoncés des résultats}

Nous pouvons à présent énoncer nos principaux résultats. Un premier théorème que nous avons obtenu se situe dans le cadre kählérien :

THÉORÈME 1. - Soit $M$ une variété kählérienne compacte connexe dont la première classe de Chern est nulle. Alors toute structure géométrique holomorphe de type algébrique affine sur $M$ est localement homogène.

L'hypothèse faite sur le type de la structure ne peut pas être évitée. Ceci devient entièrement clair si nous considérons un plongement holomorphe d'un tore algébrique dans un espace 
projectif. Ce plongement est une structure géométrique rigide non localement homogène (car non constante).

En revanche, toute application holomorphe d'une variété compacte dans une variété affine est constante et donc localement homogène en tant que structure géométrique.

Un exemple plus élaboré de structure géométrique holomorphe (de type non affine) non localement homogène sur un tore est inspiré par la construction suivante due à E. Ghys [11]. Considérons un tore $T=\mathbf{C}^{2} / \Lambda$ et supposons qu'il existe une forme linéaire $\pi: \mathbf{C}^{2} \rightarrow \mathbf{C}$ qui envoie $\Lambda$ sur un réseau $\Lambda^{\prime}$ de $\mathbf{C}$. Par abus de notation nous désignons aussi par $\pi$ la projection de $T$ sur la courbe elliptique $\mathbf{C} / \Lambda^{\prime}$. Soit $f$ une fonction méromorphe non constante sur $\mathbf{C} / \Lambda^{\prime}$; considérons sur $T$ la forme différentielle méromorphe $\tilde{\omega}=\omega+\pi^{*}(f d z)$, où $\omega$ est une forme holomorphe globale sur $T$. Il n'est pas difficile de s'assurer que $\tilde{\omega}$ est fermée et que le feuilletage holomorophe défini par $\tilde{\omega}$ en dehors de ses pôles se prolonge en un feuilletage holomorphe non singulier sur $T$ qui s'exprime en coordonnées locales :

$$
f\left(z_{1}\right) d z_{1}+d z_{2}=0
$$

Contrairement au feuilletage linéaire défini par $\omega$, le feuilletage donné par $\tilde{\omega}$ n'est pas homogène : il n'est pas invariant par les translations de $T$ dans la direction $\frac{\partial}{\partial z_{1}}$. Ceci montre que la structure géométrique $\phi$ obtenue par la juxtaposition de ce feuilletage et de la métrique plate héritée par $T$ de la métrique canonique $d z_{1}^{2}+d z_{2}^{2}$ n'est pas localement homogène.

Le corollaire 4 que nous allons voir un peu plus loin affirme que de telles structures ne peuvent exister sur des tores complexes qui n'admettent pas de fonctions méromorphes non constantes.

Remarquons aussi que la structure géométrique $\phi$ n'est pas une $G$-structure car la droite tangente au feuilletage de $\tilde{\omega}$ est isotrope (pour la métrique) en certains points de $T$ et non isotrope en d'autres.

Un complément intéressant au théorème précédent est le résultat récent de J.-M. Hwang et N. Mok qui montrent dans [17] que sur les variétés projectives unirationnelles toutes les $G$-structures d'ordre 1 holomorphes réductives et irréductibles sont plates (en particulier, localement homogènes).

En ce qui concerne les structures rigides notre résultat se précise de la manière suivante :

THÉORÈME 2. - Soit $M$ une variété kählérienne compacte connexe dont la première classe de Chern est nulle. Si $M$ possède une structure géométrique holomorphe rigide de type algébrique affine $\phi$, alors $M$ a un revêtement fini qui est un tore.

Exemple d'application : il n'existe pas de structure géométrique holomorphe rigide de type algébrique affine sur une surface $K 3$.

Ces variétés supportent en revanche des structures symplectiques holomorphes ou bien des formes volumes holomorphes [2].

Le résultat 2 généralise un théorème de $\mathrm{M}$. Inoue, S. Kobayashi et T. Ochiai [18] valable dans le cas particulier des connexions affines. Il importe de mentionner ici que la présence d'une connexion affine holomorphe ou (à plus forte raison) d'une métrique riemannienne holomorphe sur une variété kählérienne compacte entrâne automatiquement l'annulation de la première classe de Chern de la variété [18]. Le résultat de [18] règle entièrement le cas des connexions affines holomorphes et celui des métriques riemanniennes holomorphes sur les variétés kählériennes compactes : seuls les tores admettent des telles structures et celles-ci sont nécessairement localement homogènes (plates dans le cas des métriques).

Pour ce qui est des structures géométriques rigides, un théorème de M. Gromov [13] montre qu'il existe un ouvert dense $U$ de $M$ invariant par le pseudogroupe des isométries locales de $\phi$ 
dans lequel les orbites de ce pseudogroupe sont les fibres d'une fibration holomorphe localement triviale. Nous avons le résultat suivant :

THÉORÈME 3.- Soit $\phi$ une structure géométrique holomorphe rigide sur une variété complexe compacte $M$ de dimension $n$. Si p est la dimension des orbites du pseudogroupe des isométries locales de $\phi$ sur un ouvert dense invariant $U$ de $M$ et d est la dimension algébrique de $M$, alors $p+d \geqslant n$.

Pour $d=0$, nous avons le corollaire suivant qui nous permet, dans le cas particulier des tenseurs holomorphes rigides, de retrouver un résultat de F.A. Bogomolov [4].

COROLlaire 4. - Si M est une variété complexe compacte sans fonctions méromorphes non constantes, alors toute structure géométrique holomorphe rigide sur M est localement homogène en dehors d'un sous-ensemble analytique de $M$.

La suite de ce papier s'organise selon le plan suivant. La section 2 est exclusivement technique et explique le passage d'une isométrie formelle à une isométrie locale convergente. La section 3 traite du cadre kählérien et contient la preuve des théorèmes 1 et 2 . La section 4 étudie les liens entre le pseudogroupe des isométries locales d'une structure géométrique holomorphe et le corps des fonctions méromorphes de la variété. Nous y trouverons les démonstrations des théorèmes 3 et 4 . La dernière section aborde quelques conjectures et de nouvelles directions d'étude.

\section{Passage d'une isométrie formelle à une isométrie convergente}

Considérons une structure géométrique holomorphe $\phi$ sur une variété complexe $M$. Si $m, m^{\prime}$ sont deux points de $M$, le problème de l'existence d'une isométrie locale $f$ qui envoie $m$ sur $m^{\prime}$ comporte d'abord un nombre dénombrable d'obstacles algébriques : pour tout entier $s$ nous devons trouver une transformation locale $f_{s}$ qui envoie $m$ sur $m^{\prime}$ et qui préserve le $s$-jet de la structure : $J^{s}\left(f_{s}^{*} \phi\right)(m)=J^{s} \phi(m)$.

Comme la structure est d'ordre $r$, les solutions de cette équation aux dérivées partielles doivent être cherchées dans l'ensemble des $s+r$-jets de biholomorphismes locaux. Une solution de l'équation ci-dessus s'appelle un $s+r$-jet isométrique. Il s'agit $d u s+r$-jet en un point d'un biholomorphisme local de $M$ dans $M$ qui préserve le s-jet de la structure au point considéré. La question de l'existence d'un $s+r$-jet isométrique constitue un problème purement algébrique. Avoir résolu ce problème pour toutes les valeurs de $s$ apporte une solution formelle. Pour trouver une isométrie locale il faut encore s'attaquer à un problème d'analyse : trouver une solution formelle qui converge.

L'ensemble des $s+r$-jets isométriques (pour tous les couples $\left(m, m^{\prime}\right)$ ) forme un pseudogroupe pour la composition noté $I s^{s+r}$. Deux points de $M$ se trouvent dans la même orbite du pseudogroupe $I s^{s+r}$ s'il existe des systèmes de coordonnées dans lesquelles la structure géométrique admet le même développement de Taylor à l'ordre $s$, aux deux points en question.

Si le pseudogroupe des $s+r$-jets isométriques agit transitivement sur $M$, pour tout $s \in \mathbf{N}$, nous disons que la structure géométrique $\phi$ est formellement localement homogène. Ceci est équivalent au fait que le $s$-jet de la structure $\phi$ a la propriété que $\operatorname{Im} \phi^{(s)} \subset J^{s, n}(Z)$ est formée d'une seule orbite de $J^{s, n}(Z)$ sous l'action de $D^{s+r}\left(\mathbf{C}^{n}\right)$. Autrement dit, $\phi^{(s)}$ est une $G$-structure pour tout entier $s$. En particulier une structure géométrique localement homogène est nécessairement une $G$-structure.

Le but de cette section est de montrer qu'une structure géométrique holomorphe est formellement localement homogène si et seulement si elle est localement homogène. Ce résultat est une conséquence du théorème de Cartan-Kähler [6]. 
Précisons avant tout ce que nous entendons par système d'équations aux dérivées partielles. Considérons $J^{r}(N, M) \rightarrow N$ le fibré des $r$-jets d'applications d'une variété $N$ à valeurs dans une variété $M$.

DÉFInITION 2.1. - Un système d'équations aux dérivées partielles est une partie fermée $P$ de $J^{r}(N, M)$. Une solution de $P$ est une application de $N$ dans $M$ dont le $r$-jet en tout point appartient à $P$.

Pour une introduction aux notions de prolongement et d'intégrabilité formelle d'un système d'équations aux dérivées partielles nous référons à [6].

THÉORÈME 2.2 (Cartan-Kähler). - Soit $P \subset J^{r}(N, M)$ un système formellement intégrable d'équations aux dérivées partielles analytiques. Alors pour tout $p \in P$ il existe une solution locale analytique de $P$, dont le $r$-jet en un point vaut $p$.

Si $\phi$ est une structure géométrique holomorphe d'ordre $r$ sur une variété complexe $M$, nous rappelons que l'ensemble $I s^{s+r} \subset J^{s+r}(M, M)$ des $s+r$-jets isométriques est formé par les jets d'applications de $M$ dans $M$ qui préservent le $s$-jet de $\phi$. Trouver une isométrie locale de $\phi$ revient à résoudre une équation aux dérivées partielles, dans le sens qu'il faut construire un biholomorphisme local de $M$ dans $M$ tel que en tout point de son domaine de définition son $r$-jet appartient à $I s^{r}$.

Dans la preuve du lemme suivant, nous expliquons que le système d'équations aux dérivées partielles qui caractérise une isométrie d'une structure géométrique holomorphe formellement localement homogène devient formellement intégrable après un nombre fini de prolongements. La conclusion du lemme sera alors une conséquence du théorème de Cartan-Kähler.

LEMME 2.3. - Si $\phi$ est une structure géométrique holomorphe formellement localement homogène sur une variété complexe $M$, alors $\phi$ est localement homogène.

Démonstration. - Comme $\phi$ est formellement localement homogène, tous ses $s$-jets $\phi^{(s)}$ sont des $G$-structures. Nous pouvons donc identifier la structure $\phi^{(s)}$ à un sous-fibré principal du fibré des repères $R^{s+r}(M)$ de groupe structural $I s^{s+r}(m)$, le groupe $I s^{s+r}(m)$ étant formé par les $s+r$-jets isométriques qui fixent un point de $M$ (ceci ne dépend pas du point car $\phi$ est formellement localement homogène). Il s'agit d'un sous-groupe algébrique de $D^{s+r}$. Il est donc vrai que $I s^{s+r} \subset J^{s+r}(M, M)$ est une sous-variété complexe qui fibre sur $M \times M$ avec fibre $I s^{s+r}(m)$.

Considérons les images successives des projections $I s^{s+r} \rightarrow I s^{r}$, pour $s \geqslant 0$. Il s'agit d'une suite décroissante de fibrés principaux au-dessus de $M \times M$ dont les groupes structuraux sont des sous-groupes algébriques de $I s^{r}(m)$. Cette suite stabilise. Notons $F^{r}$ le fibré minimal ainsi obtenu. Il s'agit d'un sous-fibré principal de $I s^{r}$.

De manière similaire, en considérant les projections minimales sur tous les fibrés $I s^{s+r}$, nous construisons une suite de fibrés principaux

$$
\cdots \rightarrow F^{r+1} \rightarrow F^{r}
$$

qui se projettent surjectivement les uns sur les autres. Chaque fibré $F^{s+r}$ est un sous-fibré principal de $I s^{s+r}$.

Nous venons de prouver que tout $s+r$-jet isométrique se relève en un $s+r+1$-jet isométrique. Ceci est une condition plus faible que l'intégrabilité formelle du système d'équations aux dérivées partielles $\mathcal{P}$ donné par $I s^{r}$. Pour avoir l'intégrabilité formelle de $\mathcal{P}$ il suffit de démontrer que pour $s$ suffisament grand le fibré $I s^{s+r}$ représente exactement le prolongement d'ordre $s$ de $\mathcal{P}$. Ceci résulte, par exemple, d'un théorème dû à A. Grothendieck et D. Mumford [6] (chap. 
8 prop. 3.10). Nous pouvons aussi appliquer le résultat conjecturé par E. Cartan et démontré récemment par B. Malgrange [23] qui affirme que tout système différentiel analytique devient involutif au point générique après un nombre fini de prolongements.

Le théorème de Cartan-Kähler permet alors de conclure que $\phi$ est localement homogène.

\section{Variétés de Calabi-Yau}

Nous nous intéressons dans cette section aux structures géométriques holomorphes sur les variétés complexes compactes admettant une métrique de Kähler à courbure de Ricci nulle (Ricci plate). D'après les travaux de S.T. Yau [28], il s'agit précisément des variétés complexes compactes kählériennes dont la première classe de Chern est nulle, les variétés dites de CalabiYau. Pour des exemples intéressants de ce type de variétés nous référons à [2]. Dans ce contexte, notre principal résultat est le suivant :

THÉORÈME 1. - Soit M une variété kählérienne compacte connexe dont la première classe de Chern est nulle. Alors toute structure géométrique holomorphe de type algébrique affine sur $M$ est localement homogène.

La preuve se fait en deux étapes. Dans la première étape il est montré que l'existence d'une métrique de Kähler-Ricci plate sur la variété force tous les champs de tenseurs holomorphes à être parallèles, c'est-à-dire invariants par le transport parallèle de la métrique en question. Il s'agit d'une technique classique due à S. Bochner et S. Kobayashi. Il importe de mentionner ici que cette même technique a été utilisée par S. Kobayashi [19] pour prouver que le fibré tangent d'une variété complexe compacte kählérienne dont la première classe de Chern est nulle n'est pas instable dans le sens de F. Bogomolov.

THÉORÈME 3.1 (Bochner, Kobayashi). - Soit M une variété complexe compacte kählérienne munie d'une métrique de Kähler dont la courbure de Ricci est nulle. Alors tout champ de tenseurs holomorphe sur $M$ est parallèle. En particulier, un champ de tenseurs holomorphe sur $M$ qui s'annule en au moins un point est identiquement nul.

Avant d'esquisser la preuve du théorème précédent il est utile de rappeler que la présence d'une métrique kählérienne permet de considérer la dérivée covariante d'un champ de tenseurs. En particulier, si $\theta$ est un champ de tenseurs holomorphe et $X$ est un champ de vecteurs holomorphe, la dérivée covariante $\nabla_{X} \theta$ du champ $\theta$, dans la direction $X$, est un champ de tenseurs holomorphe du même type que $\theta$. L'opération précédente étant tensorielle en $X$, elle fournit un champ de tenseurs holomorphe $\nabla \theta$ qui n'est rien d'autre que la variation infinitésimale de $\theta$ par le transport parallèle (le champ de tenseurs $\theta$ est invariant par le transport parallèle de la métrique si et seulement si $\nabla \theta=0$ ).

Rappelons aussi qu'une métrique kählérienne induit des métriques hermitiennes canoniques sur tous les fibrés des tenseurs de la variété. Par la suite, la norme d'un tenseur sera prise par rapport à cette métrique hermitienne, après le choix préalable d'une métrique de Kähler.

La preuve du théorème de Bochner-Kobayashi repose sur la formule suivante, valable, dans le cas où la courbure de Ricci est nulle, pour tout champ de tenseurs $\theta$ :

$$
\Delta\left(\|\theta\|^{2}\right)=\|\nabla \theta\|^{2}
$$

$\Delta$ étant le laplacien de la métrique agissant sur les fonctions (voir [14], chap. 5).

Nous en déduisons que le laplacien de la fonction $\|\theta\|^{2}$ est en tout point positif. Or, pour toute fonction $f$ définie sur $M, \Delta f \cdot v o l$ est une forme exacte de degré maximal. Par le théorème de Stokes $\int_{M} \Delta\|\theta\|^{2} \cdot$ vol $=0$ et donc $\Delta\|\theta\|^{2}=0$. Ceci entraîne $\nabla \theta=0$. 
Dans les articles [19], [20] l'auteur utilise le théorème de Bochner pour démontrer l'inexistence de certain types de tenseurs holomorphes sur les variétés kählériennes compactes.

Le lemme suivant termine la preuve du théorème 1 :

LEMME 3.2. - Soit $M$ une variété complexe qui a la propriété que tout champ de tenseurs holomorphe sur M qui s'annule en au moins un point est identiquement nul. Alors toute structure géométrique holomorphe de type algébrique affine sur $M$ est localement homogène.

Nous faisons remarquer que le critère énoncé dans le lemme précédent est nécessaire et suffisant.

Démonstration. - La preuve se fait par l'absurde. Considérons sur $M$ une structure géométrique holomorphe d'ordre $r$ et de type algébrique affine $\phi$ qui ne soit pas localement homogène. Par définition, $\phi$ est une application holomorphe $D^{r}\left(\mathbf{C}^{n}\right)$-équivariante du fibré des $r$-repères $R^{r}(M)$ dans une $D^{r}\left(\mathbf{C}^{n}\right)$-variété affine $Z$, où $n$ désigne la dimension complexe de $M$.

D'après le lemme 2.3 la structure $\phi$ n'est pas formellement localement homogène et donc il existe un entier $s$ tel que le pseudogroupe $I s^{s+r}$ des $s+r$-jets isométriques n'est pas transitif sur $M$. Il est équivalent de dire que le $s$-jet $\phi^{(s)}: R^{s+r}(M) \rightarrow J^{s, n}(Z)$ de la structure $\phi$ est tel que l'image de $\phi^{(s)}$ dans $J^{s, n}(Z)$ contient au moins deux $D^{s+r}$-orbites.

D'après un résultat classique de théorie des invariants pour les groupes algébriques (voir, par exemple, [27]) il existe un plongement algébrique $D^{s+r}\left(\mathbf{C}^{n}\right)$-équivariant de la variété affine $J^{s, n}(Z)$ dans un espace vectoriel complexe de dimension finie $V^{(s)}$ muni d'une action algébrique linéaire du groupe $D^{s+r}\left(\mathbf{C}^{n}\right)$. Il est, par conséquent, légitime de remplacer la variété affine $J^{s, n}(Z)$ par un espace vectoriel $V^{(s)}$ muni d'une action linéaire algébrique de $D^{s+r}\left(\mathbf{C}^{n}\right)$.

Choisissons une orbite $X$ de dimension minimale parmi les orbites incluses dans l'image $\operatorname{Im} \phi^{s}$. Désignons par $\bar{X}$ l'adhérence de $X$ dans $V^{(s)}$ et par $\overline{\operatorname{Im} \phi^{(s)}}$ l'adhérence de $\operatorname{Im} \phi^{(s)}$ dans $V^{(s)}$. Il n'y a pas d'ambiguïté car dans ce cas l'adhérence dans la topologie de Zariski coïncide avec l'adhérence dans la topologie usuelle (voir [25], ch. 10).

L'ensemble $\bar{X}$ est un fermé $D^{s+r}$-invariant de $\overline{\operatorname{Im} \phi^{(s)}}$ tel que $\bar{X} \cap \operatorname{Im} \phi^{(s)} \neq \emptyset$, et $\operatorname{Im} \phi^{(s)}$ n'est pas inclus dans $\bar{X}$. La seule affirmation non triviale est la dernière. Elle est assurée par le choix de $X$ puisque, d'après un résultat fondamental de la théorie des actions algébriques ([16], 8.3), l'ensemble $\bar{X} \backslash X$ est une union d'orbites de dimension strictement inférieure à la dimension de $X$.

Soit $I$ l'idéal des fonctions régulières sur $\operatorname{Im} \phi^{(s)}$ qui s'annulent sur le fermé $\bar{X}$. Il existe dans $I$ un sous-espace vectoriel non trivial de dimension finie $D^{s+r}\left(\mathbf{C}^{n}\right)$-invariant ([16], 8.6). Considérons $\left(F_{1}, \ldots, F_{l}\right)$, une base de cet espace vectoriel et construisons le morphisme régulier $D^{s+r}\left(\mathbf{C}^{n}\right)$-invariant $T$, donné par

$$
T: v \in \overline{\operatorname{Im} \phi^{(s)}} \rightarrow\left(F_{1}(v), \ldots, F_{l}(v)\right) \in \mathbf{C}^{l} .
$$

L'application $T$ s'annule sur $\bar{X}$. La composition $T \circ \phi^{(s)}$ fournit une application holomorphe non triviale $D^{s+r}\left(\mathbf{C}^{n}\right)$-équivariante, du fibré $R^{s+r}(M)$ dans $\mathbf{C}^{l}$ avec la propriété que $0 \in \operatorname{Im}\left(T \circ \phi^{(s)}\right)$.

Montrons à présent que, quitte à modifier légèrement l'application $T$, elle descend en une application non triviale définie sur le fibré des 1-repères.

Le groupe structural du fibré des $s+r$-repères est $D^{s+r}\left(\mathbf{C}^{n}\right)$. Il existe une projection canonique $D^{s+r}\left(\mathbf{C}^{n}\right) \rightarrow D^{1}\left(\mathbf{C}^{n}\right)=G L(n, \mathbf{C})$ qui associe à chaque $s+r$-jet de biholomorphisme son 1-jet sous-jacent. Le noyau de cette projection est un sous-groupe distingué $H$.

Nous montrons à présent que $H$ est un groupe unipotent (il s'agit d'un résultat classique). Pour cela nous faisons agir $H$ fidèlement sur un espace vectoriel de dimension finie, de sorte 
que, dans une base judicieusement choisie, chaque élément de $H$ sera représenté par une matrice triangulaire supérieure dont tous les coefficients diagonaux sont égaux à 1 . En effet, le groupe $D^{s+r}\left(\mathbf{C}^{n}\right)$ agit linéairement sur l'espace vectoriel $\mathcal{H}$, des $s+r$-jets en 0 de germes de fonctions holomorphes de $\mathbf{C}^{n}$ à valeurs dans $\mathbf{C}$. Notons $\mathcal{P}_{i}$ l'espace vectoriel des polynômes homogènes en $n$ variables, de degré $i$. Alors

$$
\mathcal{H}=\mathcal{P}_{1} \oplus \cdots \oplus \mathcal{P}_{s+r}
$$

Nous considérons le drapeau de $\mathcal{H}$ formé par les sous-espaces vectoriels

$$
\mathcal{H}_{i}=\mathcal{P}_{i} \oplus \cdots \oplus \mathcal{P}_{s+r}
$$

L'action de $D^{s+r}\left(\mathbf{C}^{n}\right)$ sur $\mathcal{H}$, induit une action algébrique linéaire de $H$ sur $\mathcal{H}$, qui laisse stables les sous-espaces vectoriels $\mathcal{H}_{i}$. De plus $H$ agit trivialement sur $\mathcal{H}_{s+r}$ et sur les espaces quotients $\mathcal{H}_{i} / \mathcal{H}_{i+1}, \forall i, 1 \leqslant i \leqslant s+r-1$. Ceci montre que $H$ est unipotent.

Un résultat classique de la théorie des représentations algébriques des groupes unipotents ([16], 17.5) assure que l'action de $H \subset D^{s+r}\left(\mathbf{C}^{n}\right)$ induit sur l'espace but $\mathbf{C}^{l}$ de l'application $T \circ \phi^{(s)}: R^{s+r}(M) \rightarrow \mathbf{C}^{l}$ une filtration

$$
\mathbf{C}^{l}=V_{0} \supset V_{1} \supset \cdots \supset V_{p}=\{0\},
$$

avec la propriété que $H$ agit trivialement sur les quotients $V_{i} / V_{i+1}$, pour tout $i \in\{1, \ldots, p-1\}$.

Comme l'image $\operatorname{Im}\left(T \circ \phi^{(s)}\right)$ de l'application $T \circ \phi^{(s)}$ ne se réduit pas au point 0 , il existe au moins un indice $i \in\{1, \ldots, p-1\}$ pour lequel $\operatorname{Im}\left(T \circ \phi^{(s)}\right) \subset V_{i}$ et l'image de l'ensemble $\operatorname{Im}\left(T \circ \phi^{(s)}\right)$ par la projection $p_{i}: V_{i} \rightarrow V_{i} / V_{i+1}$ n'est pas réduite au point 0 . Alors l'application $p_{i} \circ T \circ \phi^{(s)}: R^{s+r}(M) \rightarrow V_{i} / V_{i+1}$ a une image non triviale qui contient 0 . Comme cette application est $D^{s+r}\left(\mathbf{C}^{n}\right)$-équivariante et $H$ agit par l'identité sur $V_{i} / V_{i+1}$, elle passe au quotient en une application

$$
\overline{p_{i} \circ T \circ \phi^{(s)}}: R^{1}(M) \rightarrow V_{i} / V_{i+1},
$$

$D^{s+r}\left(\mathbf{C}^{n}\right) / H=G L(n, \mathbf{C})$-équivariante. L'image de $\overline{p_{i} \circ T \circ \phi^{(s)}}$ est non triviale et contient 0.

Comme $G L(n, \mathbf{C})$ est réductif, la représentation $V_{i} / V_{i+1}$ est une somme directe de représentations irréductibles. Parmi ces représentations irréductibles il en existe au moins une dans laquelle l'image de l'application $\overline{p_{i} \circ T \circ \phi^{(s)}}$ est non triviale (et contient 0$)$. Or, toute représentation irréductible de $G L(n, \mathbf{C})$ est facteur direct d'une représentation $\left(\mathbf{C}^{n}\right)^{\otimes p} \otimes\left(\left(\mathbf{C}^{n}\right)^{*}\right)^{\otimes q}$. Ceci résulte, par exemple, de la description des représentations irréductibles du groupe $G L(n, \mathbf{C})$ [9].

L'application $G L(n, \mathbf{C})$-équivariante de $R^{1}(M)$ dans $\left(\mathbf{C}^{n}\right)^{\otimes p} \otimes\left(\left(\mathbf{C}^{n}\right)^{*}\right)^{\otimes q}$ est un champ holomorphe de tenseurs de type $(p, q)$ sur $M$ qui s'annule en au moins un point sans être identiquement nul. C'est la contradiction recherchée.

Nous démontrons maintenant le théorème 2. - La preuve s'appuie essentiellement sur un théorème de décomposition dû à A. Beauville [2] (voir aussi [22]) qui affirme que toute variété kählérienne compacte $M$ dont la première classe de Chern est nulle admet un revêtement fini $\tilde{M}$ qui est biholomorphe au produit d'un tore complexe $T$ et d'une variété kählérienne compacte simplement connexe $N$ dont la première classe de Chern est nulle.

Si la variété $M$ est munie d'une structure géométrique holomorphe rigide $\phi$, il en est de même du revêtement $\tilde{M}=T \times N$ qui hérite d'une structure rigide $\tilde{\phi}$. Pour démontrer que le facteur $N$ est trivial nous commençons par munir $N$ d'une structure géométrique holomorphe 
rigide. Nous remarquons d'abord que le fibré des $r$-repères $R^{r}(\tilde{M})$ admet $R^{r}(T) \times R^{r}(N)$ comme sous-fibré principal. Or $R^{r}(T)$ est le produit $T \times D^{r}\left(\mathbf{C}^{t}\right)$, où $t$ désigne la dimension complexe du tore $T$. Ceci implique que le fibré $R^{r}(\tilde{M})$ contient $R^{r}(N) \times T$ comme sous-fibré principal (le groupe structural admet une réduction au groupe $D^{r}\left(\mathbf{C}^{n}\right)$, où $n$ est la dimension complexe de $N)$. L'espace but étant une variété affine, la restriction de l'application $\tilde{\phi}$ au produit $R^{r}(N) \times T$ factorise en une application $\psi$ définie sur $R^{r}(N)$. Il s'agit d'une structure géométrique holomorphe rigide de type algébrique affine $\psi$ sur $N$.

La proposition suivante achève la preuve du théorème 2.

Proposition 3.3. - Il n'existe pas de structure géométrique holomorphe rigide de type algébrique affine sur les variétés kählériennes compactes simplement connexes dont la première classe de Chern est nulle.

Démonstration. - Supposons par l'absurde qu'il existe une structure géométrique $\psi$ portée par une variété complexe $N$, le couple $(N, \psi)$ ayant les propriétés requises par les hypothèses de la proposition.

D'après le théorème 1 , la structure $\psi$ est localement homogène. Un résultat classique démontré pour la première fois par K. Nomizu [26] dans le cas des métriques riemanniennes analytiques et généralisé par M. Gromov aux structures géométriques analytiques rigides [13], affirme que sur les variétés simplement connexes une isométrie locale proche de l'identité se prolonge en une isométrie globale. Par ailleurs, deux points suffisamment proches de $M$ qui sont reliés par une isométrie locale sont aussi reliés par une isométrie locale proche de l'identité (voir, par exemple, [3]).

En résumé, le groupe (de Lie complexe) des isométries globales agit transitivement sur $N$ et la variété $N$ est, par conséquent, un espace homogène. Fixons un point $x$ dans $N$ et considérons alors $X_{1}, \ldots, X_{n}$ des champs de vecteurs holomorphes sur $N$ tels que $X_{1}(x), \ldots, X_{n}(x)$ forment une base de $T_{x} N$ ( $n$ est la dimension complexe de $N$ ). Par le théorème de BochnerKobayashi le champ de tenseurs holomorphe $X_{1} \wedge \cdots \wedge X_{n}$ est partout non nul. La variété $N$ est donc une variété parallélisable quotient d'un groupe de Lie complexe $G$ par un réseau cocompact $\Gamma$. Ceci est absurde car $N$ est simplement connexe.

\subsection{Applications dans un cadre non kählérien}

Dans le travail [10], É. Ghys étudie des variétés complexes compactes obtenues par déformations des structures complexes sur les espaces homogènes de $S L(2, \mathbf{C})$.

L'idée est de considérer un réseau cocompact $\Gamma$ de $S L(2, \mathbf{C})$ et de perturber l'action par translations à droite de $\Gamma$ sur $S L(2, \mathbf{C})$ de manière que le quotient soit toujours une variété. L'auteur prouve qu'il existe des morphismes de groupe $u: \Gamma \rightarrow S L(2, \mathbf{C})$ tels que l'action à droite de $\Gamma$ sur $S L(2, \mathbf{C})$ donnée par :

$$
(m, \gamma) \in S L(2, \mathbf{C}) \times \Gamma \rightarrow u\left(\gamma^{-1}\right) m \gamma \in S L(2, \mathbf{C})
$$

soit libre et totalement discontinue. Le quotient est une variété complexe compacte $M(u, \Gamma)$ sur laquelle la métrique de Killing de $S L(2, \mathbf{C})$ induit une métrique holomorphe. Il existe des morphismes $u$ pour lesquels les variétés $M(u, \Gamma)$ ne possèdent aucun champ de vecteurs holomorphe non nul. Il est prouvé dans [10] que tout tenseur holomorphe sur une variété de type $M(u, \Gamma)$ se relève en un tenseur holomorphe sur $S L(2, \mathbf{C})$ invariant par l'action à droite de $S L(2, \mathbf{C})$ et par l'action à gauche de $u(\Gamma)$. En particulier, tout tenseur holomorphe sur $M(u, \Gamma)$ est localement homogène. Nous montrons le même résultat pour les structures géométriques d'ordre supérieur : 
PROPOSITION 3.4. - Toute structure géométrique holomorphe de type algébrique affine sur $M(u, \Gamma)$ est localement homogène.

Démonstration. - Supposons par l'absurde qu'une variété $M(u, \Gamma)$ possède une structure géométrique de type algébrique affine non localement homogène. Le lemme 3.2 implique alors l'existence d'un tenseur holomorphe sur $M(u, \Gamma)$ qui, sans être identiquement nul, s'annule en au moins un point. Ceci est absurde : ce tenseur devrait être localement homogène.

\section{Isométries locales et fonctions méromorphes}

Nous étudions ici le rapport entre la dimension des orbites du pseudogroupe des isométries locales de $\phi$ et la dimension algébrique $d$ de la variété ambiante $M$. Dans cette section nous faisons l'hypothèse supplémentaire que la structure géométrique $\phi$ est rigide. Notre résultat s'énonce de la manière suivante :

THÉORÈME 3.-Soit $\phi$ une structure géométrique holomorphe rigide sur une variété complexe compacte $M$ de dimension $n$. Si p est la dimension des orbites du pseudogroupe des isométries locales de $\phi$ sur un ouvert dense invariant $U$ de $M$ et d est la dimension algébrique de $M$ alors $p+d \geqslant n$.

Démonstration. - Considérons une structure géométrique holomorphe rigide $\phi$, d'ordre $r$ et de type $Z$. Pour tout entier positif $s$ nous considérons le $s$-jet de la structure $\phi$, donné par une application holomorphe $D^{(r+s)}\left(\mathbf{C}^{n}\right)$-équivariante $\phi^{(s)}$ de $R^{(r+s)}(M)$ dans une $D^{(r+s)}\left(\mathbf{C}^{n}\right)$ variété quasiprojective $Z^{(s)}$. La rigidité de la structure géométrique $\phi$ implique que, pour un $s$ suffisamment grand, deux points de $M$ sont dans la même orbite du pseudogroupe des isométries locales de $\phi$ si et seulement si les deux fibres de $R^{(r+s)}(M)$ situées au-dessus de ces points s'envoient par $\phi^{(s)}$ sur une même orbite de $Z^{(s)}$.

Le théorème de Rosenlicht (voir [27]) affirme qu'il existe une stratification invariante sous l'action de $D^{(r+s)}\left(\mathbf{C}^{n}\right)$

$$
Z^{(s)}=Z_{0} \supset \cdots \supset Z_{l},
$$

avec la propriété que $Z_{i+1}$ est un fermé de $Z_{i}$ et que les fonctions rationnelles sur $Z_{i}$ qui sont $D^{(r+s)}\left(\mathbf{C}^{n}\right)$-invariantes séparent les orbites de $Z_{i} \backslash Z_{i+1}$.

L'ouvert dense invariant $U$ sur lequel les orbites du pseudogroupe des isométries locales sont les fibres d'une fibration est construit de sorte que $\phi^{(s)}$ soit de rang constant sur $\left.R^{(r+s)}(M)\right|_{U}$, l'image de $\left.R^{(r+s)}(M)\right|_{U}$ par $\phi^{(s)}$ tombe dans un $Z_{i} \backslash Z_{i+1}$, tandis que l'image de $R^{(r+s)}(M)$ est incluse dans $Z_{i}$.

Considérons une petite boule $D$, de dimension complexe $n-p$, centrée en un point de $U$ et transverse aux orbites du pseudogroupe des isométries locales. Deux points $m, m^{\prime}$ de ce disque s'envoient par $\phi^{(s)}$ sur des orbites différentes de $Z_{i} \backslash Z_{i+1}$. Il existe donc une fraction rationnelle invariante $F$, sur $Z_{i}$, qui sépare ces deux orbites de $Z_{i} \backslash Z_{i+1}$. La fonction méromorphe $F \circ \phi^{(s)}$ descend en une fonction méromorphe sur $M$ qui prend des valeurs distinctes et bien définies aux points $m$ et $m^{\prime}$.

Les fonctions méromorphes sur $M$ séparent donc les points de $D$. Cela montre que le corps des fonctions méromorphes sur $M$ contient au moins $n-p$ éléments algébriquement indépendants.

Dans le cas particulier des variétés complexes qui n'admettent pas de fonctions méromorphes non constantes nous obtenons le corollaire important 4 qui affirme que toutes les structures géométriques holomorphes rigides sur ces variétés sont nécessairement localement homogènes en dehors d'un sous-ensemble analytique. 
Si nous supposons en plus que la variété est simplement connexe nous avons le corollaire suivant :

Proposition 4.1. - Soit $M$ une variété complexe compacte simplement connexe et sans fonctions méromorphes non constantes. Si M possède une structure géométrique holomorphe rigide, alors $M$ est une variété presque homogène d'un groupe de Lie complexe, dont l'orbite dense est une variété parallélisable.

Démonstration. - Soit $\phi$ une structure holomorphe rigide sur une variété $M$ complexe compacte simplement connexe et sans fonctions méromorphes non constantes. D'après le corollaire $4, \phi$ est localement homogène en dehors d'un sous-ensemble analytique de $M$.

Le théorème de prolongement des isométries locales utilisé dans la preuve de la proposition 3.3 implique que le groupe $G$ des isométries globales agit transitivement sur un ouvert dense $U \subset M$. La variété $M$ est donc un espace presque homogène du groupe $G$.

Montrons que l'orbite dense de $G$ dans $M$ est une variété parallélisable. Quitte à quotienter, il est possible de supposer que le stabilisateur d'un point de $U$ n'est pas distingué dans $G$. Désignons par $a: G \times M \rightarrow M$ l'action du groupe $G$. Nous imitons la construction de la fibration de Tits pour les variétés homogènes (voir, par exemple, [1]) et définissons, pour un point $m$ dans $U$, le noyau de l'application $d a(\cdot, m)$. En effet, si $\mathcal{G}$ est l'algèbre de Lie de $G$, vue comme l'algèbre de Lie des champs de vecteurs de $G$, invariants à droite, le noyau $\mathcal{H}_{m}$ de $d a(\cdot, m)$ est bien défini en tant que sous-algèbre de Lie de $\mathcal{G}$.

Si $m^{\prime}=g \cdot m$ pour un certain $g \in G$, alors $\mathcal{H}_{m^{\prime}}=g \cdot \mathcal{H}_{m}$, où l'action de $G$ sur les sousalgèbres de Lie de $\mathcal{G}$ se fait via la représentation adjointe de $G$ sur $\mathcal{G}$.

Supposons que $\mathcal{H}_{m}$ est de dimension $d$. Nous venons de construire une application holomorphe de $U$ dans la grassmanienne $D_{d}$ des sous-espaces vectoriels de dimension $d$ de $\mathcal{G}$. Cette application holomorphe est la restriction d'une application méromorphe de $M$ dans $D_{d}$. Comme $M$ ne possède pas de fonction méromorphe non constante, l'image dans $D_{d}$ est nécessairement formée d'un seul point. Donc l'algèbre de Lie $\mathcal{H}_{m}$ du stabilisateur de $m \in U$ est stable par la représentation adjointe.

Or nous avons supposé que le stabilisateur d'un point de $U$ n'est pas distingué dans $G$, ce qui implique que $\mathcal{H}_{m}=\{0\}$. Par conséquent, le stabilisateur d'un point de $U$ est discret.

La proposition que nous venons de démontrer admet le corollaire suivant :

Corollaire 4.2. - Si la sphère $S^{6}$ admet une structure complexe $M$, alors $M$ ne porte pas de structure géométrique holomorphe rigide.

Démonstration. - D'après [7] $M$ ne possède pas de fonction méromorphe non constante. Si, par l'absurde, $M$ admettait une structure géométrique holomorphe rigide, elle serait une variété presque homogène par la proposition précédente. Ce cas de figure est exclu par le théorème principal de l'article [15].

À présent plaçons-nous dans la situation opposée et convenons d'appeler totalement hétérogène une structure géométrique telle que les orbites génériques du pseudogroupe des isométries locales sont discrètes. Le théorème 3 montre qu'une variété complexe compacte qui porte une structure géométrique rigide totalement hétérogène possède «autant » de fonctions méromorphes algébriquement indépendantes que sa dimension : c'est une variété de Moishezon.

Contrairement au cas réel, où beaucoup de structures géométriques sont génériquement totalement hétérogènes, les seules variétés qui peuvent «loger» des structures géométriques rigides totalement hétérogènes appartiennent à une classe très particulière : les variétés de Moishezon.

Les considérations précédentes nous conduisent naturellement aux applications suivantes : 
Proposition 4.3. - Il n'existe pas de connexion affine holomorphe totalement hétérogène sur les surfaces complexes compactes.

Démonstration. - Supposons par l'absurde que la connexion affine $\nabla$ sur la surface $S$ est totalement hétérogène. Alors $S$ est nécessairement de Moishezon. Or, une surface complexe compacte est une variété de Moisheson si et seulement si elle est projective [24].

Comme la surface $S$ admet une connexion affine, sa première classe de Chern est nulle [18] et le théorème 1 implique que $\nabla$ est localement homogène.

Les arguments précédents combinés avec le théorème de Grauert-Remmert [12], qui affirme qu'une variété de Moishezon homogène compacte est projective, impliquent :

Proposition 4.4. - Il n'existe pas de connexion affine holomorphe totalement hétérogène sur les espaces homogènes compacts.

\section{Conjectures}

La conjecture générale qui se précise derrière les résultats démontrés dans cet article est la suivante :

CONJECTURE 1. - Sur les variétés complexes compactes toutes les $G$-structures holomorphes de type algébrique affine sont localement homogènes.

Pour mesurer l'étendue de cette conjecture soulignons que celle-ci implique les conjectures «faibles » suivantes :

CONJECTURE 2.- Toute métrique riemannienne holomorphe sur une variété complexe compacte est localement homogène.

CONJECTURE 3. - Toute connexion affine holomorphe sans torsion sur une variété complexe compacte est localement homogène.

Nous rappelons que la preuve des deux derniers énoncés est connue dans le cadre kählérien [18] et que sur les variétés sans fonctions méromorphes nous avons le corollaire 4.

Pour ce qui est des métriques riemanniennes holomorphes, remarquons que si l'argument de la courbure sectionnelle règle le cas des surfaces, il est en revanche impuissant en dimension supérieure. En effet, de manière similaire à la géométrie pseudo-riemannienne, la courbure sectionnelle n'est pas définie sur les plans isotropes. Elle est une fonction méromorphe, en général non constante, sur la 2-grassmannienne de la variété. Dans [8] nous avons réglé le cas de la dimension 3 sous l'hypothèse supplémentaire de simple connexité. Le cas des métriques riemanniennes holomorphes et celui des connexions affines holomorphes feront l'objet d'une publication ultérieure.

\section{RÉFÉRENCES}

[1] AKHIEZer D., Lie Group Actions in Complex Analysis, Aspects of Mathematics, 1995.

[2] Beauville A., Variétés Kähleriennes dont la première classe de Chern est nulle, J. Differential Geom. 18 (1983) 755-782.

[3] Benoist Y., Orbites des structures rigides (d'après M. Gromov), in: Feuilletages et Systèmes Intégrables (Montpellier, 1995), Birkhäuser, Boston, 1997, pp. 1-17.

[4] Bogomolov F., Classification of surfaces of class VII 0 with $b_{2}=0$, Math. USSR Izvestija 10 (2) (1976) 255-269. 
[5] Bogomolov F., Holomorphic tensors and vector bundles on projective varieties, Math. USSR Izvestija 13 (3) (1979) 499-555.

[6] Bryant R., Chern S., Gardner R., Goldschmidt H., Griffiths P., Exterior Differential Systems, M.S.R.I. Publications, Vol. 18, Springer-Verlag, 1991.

[7] Campana F., Demailly J.-P., Peternell T., The algebraic dimension of compact complex threefolds with vanishing second Betti number, Compositio Math. 112 (1) (1998) 77-91.

[8] Dumitrescu S., Structures géométriques holomorphes, Thèse E.N.S.-Lyon, 1999.

[9] Fulton W., HarRis J., Representation Theory, Graduate Texts in Mathematics, Vol. 129, SpringerVerlag, 1991.

[10] GhYs É., Déformations des structures complexes sur les espaces homogènes de $S L(2, \mathbf{C})$, J. Reine Angew. Math. 468 (1995) 113-138.

[11] GHYs É., Feuilletages holomorphes de codimension un sur les espaces homogènes complexes, Ann. Fac. Sci. Toulouse Math. (6) 5 (3) (1996) 493-519.

[12] Grauert H., REMmert R., Über kompakte homogene komplexe Mannigfaltigkeiten, Arch. Math. 13 (1962) 498-507.

[13] Gromov M., Rigid transformation groups, in: Bernard D., Choquet-Bruhat Y. (Eds.), Géométrie Différentielle, Vol. 33, Hermann, 1988, pp. 65-139.

[14] Horst C., KobaYASHI S., Topics in complex differential geometry, DMV Semin 3 (1983) 1-64.

[15] Huckleberry A., Kebekus S., Peternell T., Group actions on $S^{6}$ and complex structures on $P^{3}(\mathbf{C})$, Duke Math. J. 102 (1) (2000) 101-124.

[16] Humpreys J., Linear Algebraic Groups, Graduate Texts in Mathematics, Vol. 21, Springer-Verlag, 1975.

[17] HWANG J.-M., MoK N., Uniruled projective manifolds with irreducible reductive $G$-structure, J. Reine Angew. Math. 490 (1997) 55-64.

[18] Inoue M., Kobayashi S., Ochiai T., Holomorphic affine connections on compact complex surfaces, J. Fac. Sci. Univ. Tokyo Sect. IA Math. 27 (2) (1980) 247-264.

[19] KobaYASHI S., First Chern class and holomorphic tensor fields, Nagoya Math. J. 77 (1980) 5-11.

[20] Kobayashi S., The first Chern class and holomorphic symmetric tensor fields, J. Math. Soc. Japan 32 (2) (1980) 325-329.

[21] Kolar I., Michor P., Slovak J., Natural Operations in Differential Geometry, Springer-Verlag, 1991.

[22] LESCURE F., Sur certains espaces fibrés kaehlériens, J. Math. Pures et Appl. 57 (1978) 181-190.

[23] Malgrange B., L'involutivité générique des systèmes différentiels analytiques, C. R. Acad. Sci. Paris, Série 1326 (1998) 863-866.

[24] Moishezon B., On $n$ dimensional compact varieties with $n$ independent meromorphic functions, Amer. Math. Soc. Transl. 63 (1967) 51-77.

[25] Mumford D., Introduction to Algebraic Geometry, Harvard University, 1966.

[26] NomizU K., On local and global existence of Killing vector fields, Ann. of Math. (2) 72 (1960) 105120.

[27] Popov V., Vinberg E., Invariant theory, Algebraic Geometry 4, E.M.S. 55 (1991) 123-280.

[28] YAU S.T., On the Ricci curvature of a compact Kähler manifold and the complex Monge-Ampère equation I, Comm. Pure Appl. Math. 31 (3) (1978) 339-411.

(Manuscrit reçu le 27 janvier 2000.)

Sorin DUMitrescu

Unité de mathématiques pures et appliquées,

École normale supérieure de Lyon,

UMR 5669 du CNRS,

46, allée d'Italie,

69364 Lyon cedex 07, France

E-mail : sdumitre@umpa.ens-lyon.fr 